\title{
Harmonization of programmed cell death ligand-1 diagnostic assays in non-small cell lung cancer
}

\author{
Amy Popple, Timothy M. Illidge \\ Targeted Therapy Group, Division of Molecular and Clinical Cancer Sciences, The University of Manchester, Manchester Cancer Research Centre, \\ Manchester Academic Health Sciences Centre, Manchester, UK \\ Correspondence to: Amy Popple. Targeted Therapy Group, Division of Molecular and Clinical Cancer Sciences, The University of Manchester, The \\ Paterson Building, Wilmslow Road, Manchester, M20 4BX, UK. Email: amy.popple@ics.manchester.ac.uk. \\ Comment on: Ratcliffe MJ, Sharpe A, Midha A, et al. Agreement between Programmed Cell Death Ligand-1 Diagnostic Assays across Multiple \\ Protein Expression Cut-Offs in Non-Small Cell Lung Cancer. Clin Cancer Res 2017. [Epub ahead of print].
}

Submitted Mar 13, 2017. Accepted for publication Apr 12, 2017.

doi: $10.21037 /$ tcr.2017.04.18

View this article at: http://dx.doi.org/10.21037/tcr.2017.04.18

Targeting the programmed death 1/programmed cell death ligand-1 (PD-1/PD-L1) immune checkpoint pathway in non-small cell lung cancer (NSCLC) with anti-PD-1 and anti-PD-L1 monoclonal antibodies led to durable responses for some patients and signalled to the oncology community that immunotherapy had finally arrived as an important anti-cancer therapy $(1,2)$. Currently more than 200 clinical trials are on-going or actively recruiting targeting at least one aspect of the PD pathway in NSCLC. One of the major challenges in developing these clinical trials has been the need for standardized assays to measure tumoral PD-1 and PD-L1 expression, as a potential predictive biomarker of response $(3,4)$.

Tumor PD-L1 expression is thought to contribute to immune evasion by binding to the inhibitory receptor PD-1 on T-cells leading to T-cell "exhaustion". Interruption of the PD-1/PD-L1 signaling pathway can subsequently enhance $\mathrm{T}$-cell responses by reversing exhaustion and eliciting anti-tumor activity (5). Improved responses to antiPD-1 therapy have been seen in patients with higher PDL1 expression in NSCLC (6-8) However, the value of PD$\mathrm{L} 1$ expression in predicting patient responses to anti-PD-1/ PD-L1 treatment remains controversial (9). The results using PD-L1 as a predictive biomarker for patient selection have been conflicting, in part, due to heterogeneity in PDL1 expression and may be further exacerbated by the use of independent PD-L1 immunohistochemistry (IHC) assays, for anti-PD-1 mAbs Nivolumab and Pembrolizumab in clinical trials (10). Nivolumab clinical trials in NSCLC utilized the Dako 28-8 anti-PD-L1 antibody to identify three PD-L1 thresholds $(\geq 1 \%, \geq 5 \%$ and $\geq 10 \%$ of tumor cells) (7); whereas PD-L1 biomarker measurements in Pembrolizumab trials are calculated using the Dako 22C3 clone with two thresholds ( $\geq 1 \%$ and $\geq 50 \%$ ) (11). The clinical activity of Nivolumab in a phase II trial appeared independent of tumoral PD-L1 expression as clinical responses were seen in PD-L1 negative patients. In contrast, Pembolizumab treatment appeared to correlate with tumor cell expression of PD-L1 in over $50 \%$ of cells. Durvalumab, an anti-PD-L1 monoclonal antibody, uses the Ventana platform (Ventana SP263) as a diagnostic test for PD-L1 expression (12). The Ventana platform (SP142) is used with Atezolizumab, another anti-PD-L1 antibody. While the threshold for SP263 is set at $\geq 25 \%$ of PD-L1 positive tumor cells, SP142 also takes into account PD-L1 expression of immune cells resulting in a more complicated grading system (TC0-3 and IC0-3) (13).

Radcliffe and colleagues have recently compared the use of three commercially available anti-PD-L1 IHC diagnostic assays in NSCLC (Dako 28-8, Dako 22C3 and Ventana SP263) (14). The main objective was to establish confidence in PD-L1 expression on patient tumor samples measured by these independent assays to enable flexibility in assay selection between laboratories and for comparison of results across clinical trials. The authors have provided analysis for PD-L1 expression on the largest cohort of NSCLC patient 
samples to date, 493 samples were cut into consecutive sections and evaluated by each of the antibody clones. All samples were assessed by the same pathologist and 200 of which were independently reviewed by a second pathologist.

The main important finding was the high concordance across PD-L1 assay platforms. The authors found correlative expression patterns of tumor membrane PD-L1 with an overall percentage agreement (OPA) of $>90 \%$ recorded across assays and between variable expression level thresholds. OPA increased with higher expression cut-off levels, $91 \%$ at $\geq 1 \%$ up to $95 \%$ at $\geq 50 \%$ tumor membrane positivity. To fully compare the different antiPD-L1 antibodies the group altered the scoring thresholds for the Ventana SP263 assay to include lower thresholds below $10 \%$ PD-L1 positive tumor cells. The SP263 and $22 \mathrm{C} 3$ assays showed a higher level of concordance at expression levels above $50 \%$ while a $25 \%$ positivity threshold for SP263 was equivalent to a $10 \%$ threshold with the Dako 28-8. These data highlight the importance of appropriate and comparable threshold values of PD-L1 expression. Thresholds should be calculated rigorously to ensure the largest percentage of patients show clinical efficacy and values need to be flexible depending on the type of combinational therapies tested. One aspect not currently addressed by the authors was whether assay agreement was independent of, or correlates with, patient tumor stage. Much of the patient demographic was disease stage I and II (80\%), whereas collection of higher numbers of samples from stage III and IV patients would enable this to be investigated further. To fully harmonize PD-L1 assay platforms between clinical sites and across trials the reproducibility must be maintained at all disease stages.

Other studies have also sought to compare the use of anti-PD-L1 antibodies. Gaule et al. (15) examined whether 6 antibodies (SP142, E1L3N, 9A11, SP263, 22C3 and 28-8) could be used interchangeably to detect PD-L1 on an index tissue microarray (TMA). The study concluded that antibody clones could be used interchangeably to detect PD-L1 but that within tumor tissue samples inherent heterogeneity of PD-L1 expression increases assay variability across studies. This technical challenge was addressed by Ratcliffe $e t a l$. by serial sectioning of the same tissue biopsy for each patient for use across separate assay platforms.

In addition to identifying the interchangeable use of independent anti-PD-L1 assay platforms an advantage would be to be able to substitute different antibody clones within the same assay detection platform. Neuman et al. investigated the use of the Dako clone 22C3 by IHC staining on the Ventana detection platform (16). Forty one cases of NSCLC were independently evaluated by two pathologists for PD-L1 expression and the same results were obtained by both platforms for 35 samples. Low interand intra-observer variation was recorded and the study concluded that the Dako 22C3 antibody clone could be used in facilities housing the Ventana platform.

In this analysis the authors compared three well validated diagnostic assays. Potentially there is greater value in assessing other commercially available, less well validated, antibodies such as E1L3N or the Ventana SP142. E1L3N (Cell Signaling Technologies) anti-PD-L1 antibody has recently been directly compared to Ventana SP263 (17) on a TMA containing 100 formalin fixed paraffin embedded (FFPE) NSCLC cores. The cores were analysed by two pathologists in a blinded study and PD-L1 staining patterns showed that the SP263 assay was more sensitive and had a wider dynamic range than the E1L3N assay. This type of research is useful to develop increasing access to PD-L1 staining techniques.

An industrial-academic collaboration is already underway with the aim to standardise PD-L1 IHC assays used in clinical trials (18). The Blueprint PD-L1 IHC assay comparison project recently published results from Phase 1 of the project where 39 NSCLC tissue samples were stained with anti-PD-L1 antibody clones 22C3, 28-8, SP142 and SP263. Although only in a small number of samples; importantly, PD-L1 expression was scored on both tumor and immune cells at any intensity. Variability across assays was increased for immune cells positivity than for tumor cell expression. The 22C3, 28-8 and SP263 were all comparable assays, as confirmed here by Ratcliffe et al. whereas the Ventana SP142 antibody exhibited fewer positively stained cells than the other clones.

Interestingly, Ratcliffe et al. found the variability was higher between two scoring pathologists than across IHC assays by a single scorer. The variability between scorers increased at PD-L1 expression levels below $10 \%$ of the tissue with an OPA between the original study pathologist and the independent pathologist decreasing from $95 \%$ at a cut-off level of $\geq 50 \%$ down to $76 \%$ at $\geq 1 \%$. The authors assign this to the scorers' perception of expression levels. This is an important finding as patients whose tumors have been categorized as PD-L1 negative continue to respond to anti-PD-1 therapies and therefore any incidence of a false negatives need to be ruled out. PD-L1 on other immune cells, PD-L2 expression and even the presence of PD-1 
expressing immune cells may all be contributing factors in increasing confidence in biomarkers for therapies targeting the PD pathway. Calles et al. investigated the expression of PD-1 and both ligands, PD-L1 and PD-L2, in 114 patients with KRAS-mutant NSCLC (19). PD-L1 expression, but not PD-L2, was associated with smoking status. Another novel approach, by Paulsen et al., measured stromal PD-1 and PD-L1 in 536 NSCLC patient samples (20). PD-L1 positive immune cells within the stromal compartment and intraepithelial tumor-infiltrating lymphocytes expressing PD-1 were identified as independent positive prognostic factors in NSCLC.

Overall this research draws attention to the importance of graded PD-L1 positivity scores for combinational therapies. PD-L1 expression doesn't conform to established predictive biomarkers where a beneficial treatment effect is expected between biomarker positive and negative patients. PD-L1 expression is heterogeneous and alters over time during an evolving immune response depending on concurrent or prior treatments such as chemotherapy and radiotherapy. As a result, challenges arise when using patient archival tissue samples. PD-L1 negative patients can also benefit from, and show responses to, anti-PD-1/ PD-L1 therapies. More detailed analysis of the tumor microenvironment may provide answers in these PD-L1 negative patients. Indeed, localization of PD-L1/PD-1 within tumor compartments adds to the complexity of its use as a biomarker. To conclude, these data suggest harmonization in PD-L1 assays is achievable with the caveat that additional scorers, with sufficient training, rather than the choice of antibody clone would provide higher assay validity and reproducibility across laboratories.

\section{Acknowledgments}

Funding: None.

\section{Footnote}

Provenance and Peer Review: This article was commissioned and reviewed by the Section Editor Wei Xu (Division of Respiratory Disease, Department of Geriatrics, the First Affiliated Hospital of Nanjing Medical University, Nanjing, China).

Conflicts of Interest: Both authors have completed the ICMJE uniform disclosure form (available at http://dx.doi. org/10.21037/tcr.2017.04.18). The authors have no conflicts of interest to declare.

Ethical Statement: The authors are accountable for all aspects of the work in ensuring that questions related to the accuracy or integrity of any part of the work are appropriately investigated and resolved.

Open Access Statement: This is an Open Access article distributed in accordance with the Creative Commons Attribution-NonCommercial-NoDerivs 4.0 International License (CC BY-NC-ND 4.0), which permits the noncommercial replication and distribution of the article with the strict proviso that no changes or edits are made and the original work is properly cited (including links to both the formal publication through the relevant DOI and the license). See: https://creativecommons.org/licenses/by-nc-nd/4.0/.

\section{References}

1. Topalian SL, Hodi FS, Brahmer JR, et al. Safety, activity, and immune correlates of anti-PD-1 antibody in cancer. $\mathrm{N}$ Engl J Med 2012;366:2443-54.

2. Brahmer JR, Tykodi SS, Chow LQ, et al. Safety and activity of anti-PD-L1 antibody in patients with advanced cancer. N Engl J Med 2012;366:2455-65.

3. Rebelatto MC, Midha A, Mistry A, et al. Development of a programmed cell death ligand-1 immunohistochemical assay validated for analysis of non-small cell lung cancer and head and neck squamous cell carcinoma. Diagn Pathol 2016;11:95.

4. Cree IA, Booton R, Cane P, et al. PD-L1 testing for lung cancer in the UK: recognizing the challenges for implementation. Histopathology 2016;69:177-86.

5. Jiang Y, Li Y, Zhu B. T-cell exhaustion in the tumor microenvironment. Cell Death Dis 2015;6:e1792.

6. Taube JM, Klein A, Brahmer JR, et al. Association of PD1, PD-1 ligands, and other features of the tumor immune microenvironment with response to anti-PD-1 therapy. Clin Cancer Res 2014;20:5064-74.

7. Rizvi NA, Mazieres J, Planchard D, et al. Activity and safety of nivolumab, an anti-PD-1 immune checkpoint inhibitor, for patients with advanced, refractory squamous non-small-cell lung cancer (CheckMate 063): a phase 2, single-arm trial. Lancet Oncol 2015;16:257-65.

8. Carbognin L, Pilotto S, Milella M, et al. Differential Activity of Nivolumab, Pembrolizumab and MPDL3280A according to the Tumor Expression of Programmed Death-Ligand-1 (PD-L1): Sensitivity Analysis of Trials in 
Melanoma, Lung and Genitourinary Cancers. PLoS One 2015;10:e0130142.

9. Grigg C, Rizvi NA. PD-L1 biomarker testing for nonsmall cell lung cancer: truth or fiction? J Immunother Cancer 2016;4:48.

10. Kerr KM, Nicolson MC. Non-Small Cell Lung Cancer, PD-L1, and the Pathologist. Arch Pathol Lab Med 2016;140:249-54.

11. Daud AI, Wolchok JD, Robert C, et al. Programmed Death-Ligand 1 Expression and Response to the AntiProgrammed Death 1 Antibody Pembrolizumab in Melanoma. J Clin Oncol 2016;34:4102-9.

12. Planchard D, Yokoi T, McCleod MJ, et al. A Phase III Study of Durvalumab (MEDI4736) With or Without Tremelimumab for Previously Treated Patients With Advanced NSCLC: Rationale and Protocol Design of the ARCTIC Study. Clin Lung Cancer 2016;17:232-6 e1.

13. Fehrenbacher L, Spira A, Ballinger M, et al. Atezolizumab versus docetaxel for patients with previously treated non-small-cell lung cancer (POPLAR): a multicentre, open-label, phase 2 randomised controlled trial. Lancet 2016;387:1837-46.

14. Ratcliffe MJ, Sharpe A, Midha A, et al. Agreement between Programmed Cell Death Ligand-1 Diagnostic

Cite this article as: Popple A, Illidge TM. Harmonization of programmed cell death ligand-1 diagnostic assays in non-small cell lung cancer. Transl Cancer Res 2017;6(Suppl 3):S553-S556. doi: $10.21037 /$ tcr.2017.04.18
Assays across Multiple Protein Expression Cut-Offs in Non-Small Cell Lung Cancer. Clin Cancer Res 2017.

15. Gaule P, Smithy JW, Toki M, et al. A Quantitative Comparison of Antibodies to Programmed Cell Death 1 Ligand 1. JAMA Oncol 2016.

16. Neuman T, London M, Kania-Almog J, et al. A Harmonization Study for the Use of 22C3 PD-L1 Immunohistochemical Staining on Ventana's Platform. J Thorac Oncol 2016;11:1863-8.

17. Smith J, Robida MD, Acosta K, et al. Quantitative and qualitative characterization of Two PD-L1 clones: SP263 and E1L3N. Diagn Pathol 2016;11:44.

18. Hirsch FR, McElhinny A, Stanforth D, et al. PDL1 Immunohistochemistry Assays for Lung Cancer: Results from Phase 1 of the Blueprint PD-L1 IHC Assay Comparison Project. J Thorac Oncol 2017;12:208-22.

19. Calles A, Liao X, Sholl LM, et al. Expression of PD-1 and Its Ligands, PD-L1 and PD-L2, in Smokers and Never Smokers with KRAS-Mutant Lung Cancer. J Thorac Oncol 2015;10:1726-35.

20. Paulsen EE, Kilvaer TK, Khanehkenari MR, et al. Assessing PDL-1 and PD-1 in Non-Small Cell Lung Cancer: A Novel Immunoscore Approach. Clin Lung Cancer 2017;18:220-233.e8. 